\title{
Studies on Pedestrian Fundamental Diagrams: Field Observations and Controlled Experiments
}

\author{
Jyoti Biraj Das, Ujjal Chattaraj, and Sonali Nayak
}

\begin{abstract}
The simplest form of transportation is walking and it is one of the basic mobility. To understand pedestrian dynamics a number of empirical studies and a series of experiments have been done earlier. Prediction of pedestrian movements is valuable in many contexts as pedestrians are the most vulnerable road users. Under different situations pedestrians behave differently. The required data are collected from both field and experimental analysis considering mix gender pedestrian conditions. This study was conducted in two phases, in the first phase, we conducted the study and the related research works on pedestrian movement i.e. collection of field data and its representation of fundamental diagrams. In the second phase, the pedestrian motion was analyzed under experimental observation which was conducted under ideal conditions, the absence of uncontrollable disturbing factors such as side by side pedestrian movement, tail back effect and overtaking etc. The experimental set up for simple single file pedestrian flow with boundary condition was done in which various disturbing factors are avoided in the experimental studies. Fundamental diagrams of speed-density, speed-flow, flow-density and distance headway-speed were compared with the data collected from field observations. Differences of pedestrian flow in between field observation and conducted experimental observation are studied through hypothesis testing.
\end{abstract}

Index Terms - Boundary condition, fundamental diagram, hypothesis test, pedestrian dynamics

\section{INTRODUCTION}

In India the level of versatility in urban and in provincial areas has expanded because of the creation of more mechanized and non-mechanized vehicles. Most of the travel has been proficient by the vehicles yet there are sure places where we need to stroll to achieve our destination. The most fundamental and primary form of human mobility is walking, which is a vital factor for the progress towards human civilization. Human's first mean of transportation is walking. Most of the trips by other type of modes may either begin or end with walking. Although motorized transportation has priority over all other types of transportation systems, but pedestrian flow plays an important consideration in many areas like planning of urban systems, traffic forecasting and operations, land use planning, designing of important public architectures. Better mobility can be provided if there is clear understanding of pedestrian flow. In case of vehicular traffic, separate directions are provided for

Jyoti Biraj Das, Research Scholar, Department of Civil Engineering, National Institute of Technology, Rourkela, India,769008

Ujjal Chattaraj, Assistant Professor, Department of Civil Engineering, National Institute of Technology, Rourkela, India, 769008

Sonali Nayak, PG Student, Department of Civil Engineering, National Institute of Technology, Rourkela, India,769008 vehicles by lanes and flow is regulated, whereas pedestrian move in multi direction depending up on their own choice and purpose of the trip etc. The pedestrian flow can be unidirectional or single file motion (where pedestrian movement is in one direction, space between pedestrians in the direction of motion affects pedestrian speed) or bi-directional (where direction of movement is in both directions). Pedestrian movement is highly vulnerable, chaotic and complex in nature. A pedestrian tries to have a most convenient way for movement, So that delays can be minimized by avoiding obstacles and other passing by pedestrians, meant to use an optimal path to attain the destination at a certain time.

\section{LITERATURE REVIEW}

Researches formed on pedestrian dynamics has given valuable knowledge to designer of public facility and these studies have helped in better designing of facilities with safety and crowd management. The better understanding of factors affecting pedestrian dynamics requirement increases with the appearance of new well designed public facilities. Based on empirical data several studies on pedestrian flow have been performed to obtain the quantitative analysis of pedestrian flow characteristics. Mostly used observables are speed, flow and density. Many researchers have performed several studies and proposed statistical relationship between these pedestrian flow characteristics which is called as fundamental diagram, which is a major input for designing and planning of pedestrian facilities. Empirically pedestrian crowds have been studied from three decades (Hankin and Wright (1958), Oeding (1963), Hoel (1968), Older (1968) Navin and Wheeler (1969), Fruin(1971) Weidmann (1993), Seyfried et al.(2005) and Helbing et al. (2007)). The procedure of data collection is different in each study. For designing and safety of any existing or proposed facilities the long accepted data, formulas with regulations, standards and handbooks are followed. Designing for Pedestrians: A Level of Service Concept (1970) and subsequent books, Pedestrian Planning and Design (Fruin, 1971, 1987) are followed as a prime source of data.

\section{EXPERIMENTS}

This section describes experimental and field study, which were designed for the development of the fundamental diagram. The laboratory experiments which conducted in N.I.T Rourkela campus are similar to the experiments which were conducted for the development of a fundamental diagram of the characteristics for German pedestrians in Seyfried et al. (2005) and Chattaraj et al. (2009). The shape and size, which are mentioned in Chattaraj et al. (2009) is adopted in this laboratory experiment. The 
corridor was set up with length $17.3 \mathrm{~m}$ and width $2 \mathrm{~m}$ framed with chairs and ropes, where entry and exit were demarcated with two ranging rods. Within this measured experimental section gender mix single file pedestrian motion were studied. The groups of pedestrians were asked to walk through the prepared corridor without overtaking, interaction and other disturbing actors. Rudimentary data recorded with the help of photographic procedure of video recording.

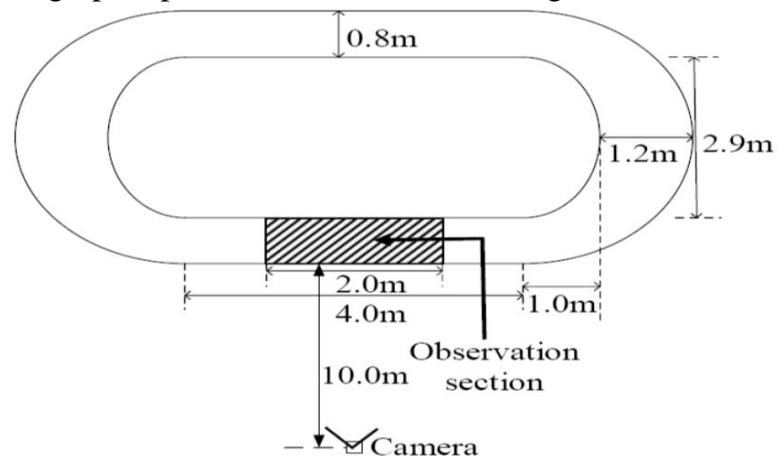

Fig. 1 Experimental setup followed in the single file pedestrian experimental observation

Besides the experimental studies, a field study has been done at LA Hall inside NIT, Campus. The data were collected with respect to mix gender. The primary aim of this data collection was to observe the variation occurring in pedestrian motion characteristics. Fundamental information recorded by a photographic method of feature recording with the length $17.3 \mathrm{~m}$ and width $2 \mathrm{~m}$ of the segment. The video camera was set was situated with an available length from the considered area. In field study area the pedestrian motion is unidirectional.

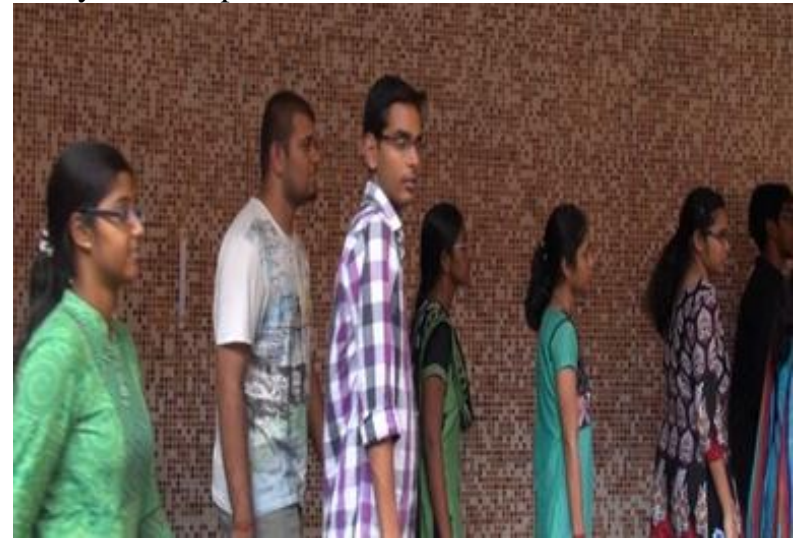

Fig. 2 Field setup followed in the single file pedestrian motion

\section{Data Collection And Data Decoding}

Through the video recording the pedestrian movement is recorded. The entry and exit time for each pedestrians can be manually recorded from the video. In an experimental study the entry and exit were marked with the ranging rods, where as in field study the entry, exit and the outline of the section was marked properly with the chalk powder and tape. The difference between the entry and exit time gives the crossing time for each pedestrian. By acquiring the intersection time of every walker the speed rate can be computed. If Tin is the entry time and Tout is the exit time, where Lo is the total length of the section, then speed $(\mathrm{Vp})$ can measured by the formula given below.

$$
\mathrm{Vp}=\mathrm{Lo} /(\text { Tout-Tin) }
$$

After Finding the speeds for each pedestrian, the flow per second is manually counted. Flow characterizes the quantity of passer by going in the watched or considered area every second. Speed, flow and density are interrelated with each other. By finding speed and flow, the density can be found out,From the equation of equilibrium given below

$$
\mathrm{Q}=\mathrm{v} \cdot \mathrm{k}
$$

Density is the no of pedestrians passing per unit length. If the density is denoted by $\mathrm{k}$ and 10 is the unit length and $\mathrm{Nf}$ is the no of pedestrians passing by, then density can be given by following equation

$$
\mathrm{K}=\mathrm{Nf} / 10
$$

Flow is the no of pedestrians passing certain length in certain duration by calculating speed and density, flow can be measured from the equilibrium equation shown above.

\section{RESULTS AND DISCUSSIONS}

This section shows the collected data analysis and also the acquired results obtained from the study. The first part of the result represents the fundamental relation between the pedestrian dynamics entities. The variations between the relationships of different pedestrian characteristics are studied through the fundamental diagrams. The primary focus is to study the speed-density, flow-density, speed-flow and distance headway-speed relationship for gender mix group under experimental and field observations. The second part of this section gives the comparison results obtained from the Hypothesis tests for different data sets.

Followings are the fundamental diagram for pedestrian mixed gender movement (LA Hall).

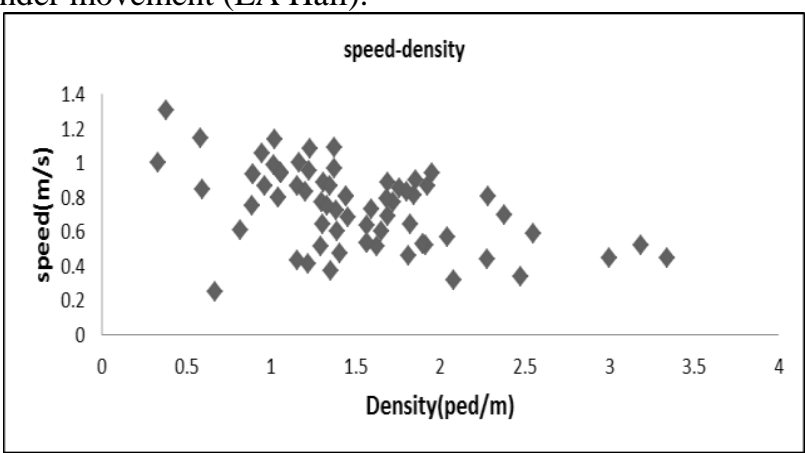

Fig. 3 Speed-Density plot for mixed gender

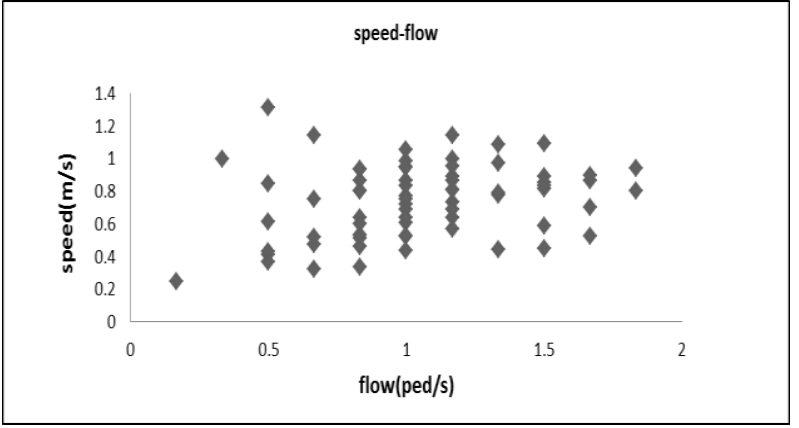

Fig. 4 Speed-Flow plot for mixed gender 


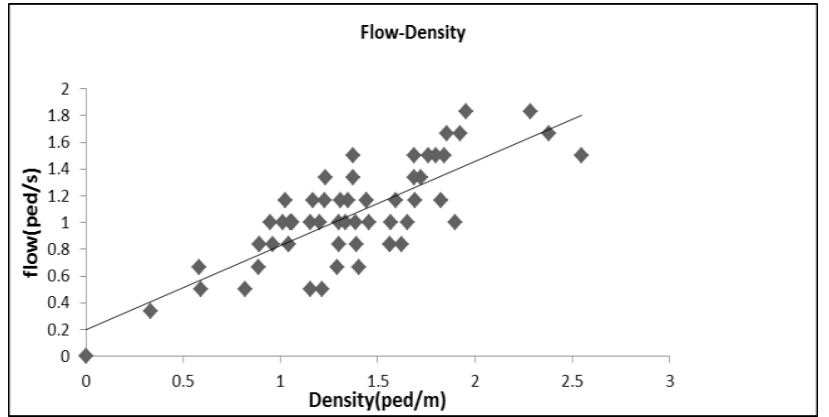

Fig. 5 Flow-Density Plot For Mixed Gender

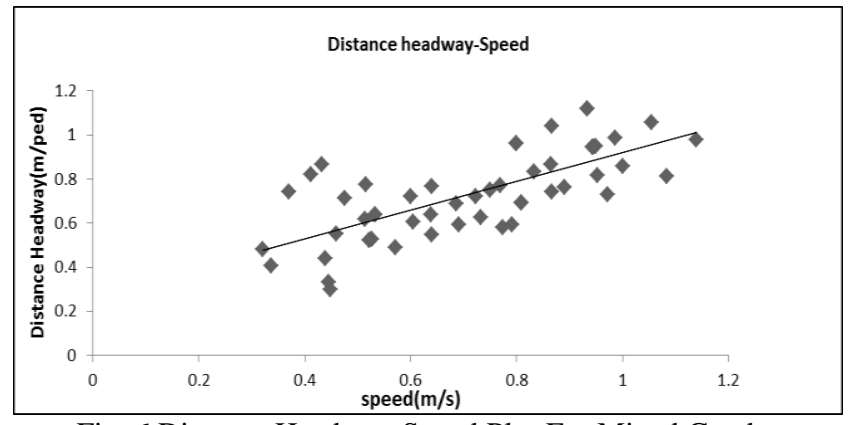

Fig. 6 Distance Headway-Speed Plot For Mixed Gender

Hypothesis comparison between field and experimental studies

TABLE I

Z-TEST: Two SAMPLE FOR MEANS MIXED GENDER SPEED

\begin{tabular}{lcc}
\hline \hline Componets & Variable 1 & Variable 2 \\
\hline Mean & 0.745 & 0.597 \\
Variance & 0.077 & 0.094 \\
\hline \hline Observations & 357 & 443
\end{tabular}

In the above $\mathrm{z}$ test for speed the mean values for variable 1 and variable 2 are $0.75 \mathrm{~m} / \mathrm{s}$ and $0.6 \mathrm{~m} / \mathrm{s}$. The two data sets for speed between field and experimental study are considered to be different. The mean speed value obtained is considered as significant.

TABLE II

Z-TEST: TWo SAMPLE FOR MEANS MIXED GENDER DISTANCE HEADWAY

\begin{tabular}{lcc}
\hline \hline Componets & Variable 1 & Variable 2 \\
\hline Mean & 0.802 & 0.337 \\
Variance & 0.196 & 0.012 \\
\hline \hline Observations & 357 & 443
\end{tabular}

In the above $\mathrm{z}$ test for distance headway the mean values for variable 1 and variable 2 are $0.8 \mathrm{~m}$ and $0.34 \mathrm{~m}$. The two data sets for distance headway between field and experimental study are considered to be different. The mean distance headway value obtained is considered as extremely significant.

\section{SUMMARY AND CONCLUSION}

This study was conducted to visualize the difference between experimental and field study. The experimental study was done with different pedestrians group for single file movement. Field study is done with respect to mixed gender criteria in this study. Speed, density and flow were manually calculated and the relationship between different pedestrian's characteristics is established for each field study through fundamental diagram. The difference which is existing between the field and experimental study are evaluated by statically hypothesis testing with respect to mixed gender. The significance of each comparison is shown.

In this research, comparisons between experimental and different field observations were done with respect to mixed gender conditions. The comparison for speed and distance headway for mixed gender pedestrians between field (LA Hall, NIT campus) and experimental study (N.I.T campus) were found to be extremely significant. It is found from the hypothesis that for speed the difference is marginal significant and for distance head way the difference was found to be extremely significant.

\section{REFERENCES}

[1] Chattaraj, U., Seyfried, A. and Chakroborty, P. (2009). Comparison of Pedestrian Fundamental Diagram Across Cultures. Advances in Complex systems, 12(3), pp. 393-405. http://dx.doi.org/10.1142/S0219525909002209

[2] Chattaraj, U., Chakroborty, P. and Seyfried, A. (2010). Empirical Studies on Pedestrian Motion Through Corridors of Different Geometries. In Proceedings of the 89th Annual Meeting of the Transportation Research Board, Washington, D.C, USA.

[3] Chattaraj, U. (2011). Understanding Pedestrian Motion: Experiments and Modelling. Ph.D. thesis. IIT Kanpur, India.

[4] Chattaraj U., Seyfried A., Chakroborty P.,Biswal M. (2013a). Modelling single file pedestrian motion across cultures. Procedia Social and Behavioral Sciences (104), Elsevier, Agra, India, pp. 698-707.

[5] Chattaraj U., Chakroborty P., Arumuga S. N. (2013b). Empirical Studies on Impacts of Obstacle inside Corridor on Pedestrian Flow. Procedia Social and Behavioral Sciences (104), Elsevier, Agra, India, pp. 668-677.

[6] Chattaraj, U., Chakroborty, P. and Seyfried, A. (2013c). Some Empirical Studies on Evacuation from Halls. In Proceedings of the 9th Conference on Traffic and Granular Flow, Springer, Moscow, Russia, pp. 207-216. http://dx.doi.org/10.1007/978-3-642-39669-4_21

[7] Hankin, B.D. and Wright, R.A. (1958). Passenger Flow in Subways. Operational Research Quarterly, 9(2), pp. 81-88. http://dx.doi.org/10.1057/jors.1958.9

[8] Helbing, D., Bunza, L., Johansson, A. and Werner, T. (2005). Self Organized Pedestrian Crowd Dynamics: Experiments, Simulations and Design Solutions. Transportation Science, 39(1), pp. 1-24. http://dx.doi.org/10.1287/trsc. 1040.0108

[9] Helbing, D., Johansson, A. and Al-Abideen, H.Z. (2007). Dynamics of Crowd Disasters: An Empirical Study. Physical Review E, 75(4), pp. 046109 (1-7). http://dx.doi.org/10.1103/PhysRevE.75.046109

[10] Henderson, L.F. and Lyons, D.J. (1972). Sexual Differences in Human Crowd Motion. Nature, 240(5380), pp. 353-355. http://dx.doi.org/10.1038/240353a0

[11] Hoogendoorn, S.P. and Daamen, W. (2004). Self-Organization in Walker Experiments. In Proceedings of the 5th Symposium on Traffic and Granular Flow, (Eds.: Hoogendoorn, S.P., Luding, S., Bovy, P.H.L., Schreckenberg, M. and Wolf, D.E.), Springer, Delft, The Netherlands, pp. $121-132$.

[12] Hoogendoorn, S.P. and Daamen, W. (2005). Pedestrian Behavior at Bottlenecks. Transportation Science, 39(2), pp. 147-159. http://dx.doi.org/10.1287/trsc. 1040.0102

[13] Isobe, M., Adachi, T. and Nagatani, T. (2004). Experiment and Simulation of Pedestrian Counter Flow. Physica A, 336(3-4), pp. 638-650. http://dx.doi.org/10.1016/j.physa.2004.01.043

[14] Kretz, T., Gruenebohm, A., Kaufman, M., Mazur, F. and Schreckenberg, M. (2006). Experimental Study of Pedestrian Counterflow in a Corridor. Journal of Statistical Mechanics: Theory and Experiment, P10001. http://dx.doi.org/10.1088/1742-5468/2006/10/P10014 
[15] Mori, M. and Tsukaguchi, H. (1987). A New Method for Evaluation of Level of Service in Pedestrian Facilities. Transportation Research Part A, 21A(3), pp. 223-234. http://dx.doi.org/10.1016/0191-2607(87)90016-1

[16] Morrall, J.F., Ratnayake, L.L. and Seneviratne, P.N. (1991). Comparison of CBD Pedestrian Characteristics in Canada and Sri Lanka. Transportation Research Record, 1294, Transportation Research Board, National Research Council, Washington, DC, USA, pp. 57-61.

[17] Navin, F.P.D. and Wheeler, R.J. (1969). Pedestrian Flow Characteristics. Traffic Engineering, 39(9), pp. 30-36.

[18] Older, S.J. (1968). Movement of Pedestrians on Footways in Shopping Streets. Traffic Engineering and Control , 10(4), pp. 160-163.

[19] Polus, A., Joseph, J.L. and Ushpiz, A. (1983). Pedestrian Flow and Level of Service. Journal of Transportation Engineering, ASCE, 109(1), pp. 46-56.

http://dx.doi.org/10.1061/(ASCE)0733-947X(1983)109: $1(46)$

[20] Rastogi, R., Ilango, T. and Chandra, S. (2011a). Design Implications of Walking Speed for Pedestrian Facilities, Journal of Transportation Engineering, ASCE, 137(10), pp. 687-696. http://dx.doi.org/10.1061/(ASCE)TE.1943-5436.000025 1

[21] Rastogi, R., Chandra, S., Vamsheedhar, J. and Das, V. (2011b) Parametric study of pedestrian speeds at midblock crossings. Journal of Urban Planning and Development, ASCE, 137(4), pp. 381-389. http://dx.doi.org/10.1061/(ASCE)UP.1943-5444.000008 3

[22] Sahani R., Bhuyan P. (2013). Level of Service Criteria of off-street Pedestrian Facilities in Indian Context using Affinity Propagation Clustering. Procedia Social and Behavioral Sciences (104), Elsevier, Agra, India, pp. 718-727. http://dx.doi.org/10.1016/j.sbspro.2013.11.166

[23] Seyfried, A., Steffen, B., Klingsch, W. and Boltes, M. (2005). The Fundamental Diagram of Pedestrian Movement Revisited. Journal of Statistical Mechanics: Theory and Experiment, P10002. http://dx.doi.org/10.1088/1742-5468/2005/10/P10002

[24] Seyfried, A., Passon, O., Steffen, B., Boltes, M., Rupprecht, T. and Klingsch, W. (2009). New Insights into Pedestrian Flow through Bottlenecks. Transportation Science, 43(3), pp. 395-406. http://dx.doi.org/10.1287/trsc. 1090.0263

[25] Young, S.B. (1999). Evaluation of Pedestrian Walking Speeds in Airport Terminals. Transportation Research Record, 1674, Transportation Research Board, National Research Council, Washington, DC, USA, pp. 20-26.

http://dx.doi.org/10.3141/1674-03 